In Situ

Revue des patrimoines
In Situ

Revue des patrimoines

29 | 2016

Ensembles mobiliers, industriels, techniques.

Connaissance, protection, conservation, présentation au public

\title{
La maison atelier de Foujita à Villiers-le-Bâcle
}

The house-workshop of Foujita in Villiers-le-Bâcle

\section{Anne Le Diberder}

\section{(2) OpenEdition}

\section{Journals}

Édition électronique

URL : http://journals.openedition.org/insitu/13846

DOI : 10.4000/insitu. 13846

ISSN : 1630-7305

Éditeur

Ministère de la culture

Référence électronique

Anne Le Diberder, "La maison atelier de Foujita à Villiers-le-Bâcle », In Situ [En ligne], 29 | 2016, mis en ligne le 05 octobre 2016, consulté le 19 avril 2019. URL : http://journals.openedition.org/insitu/13846 DOI : 10.4000/insitu. 13846

Ce document a été généré automatiquement le 19 avril 2019

\section{c) (i) $९$}

In Situ Revues des patrimoines est mis à disposition selon les termes de la licence Creative Commons Attribution - Pas d'Utilisation Commerciale - Pas de Modification 4.0 International. 


\section{La maison atelier de Foujita à Villiers-le-Bâcle}

The house-workshop of Foujita in Villiers-le-Bâcle

\section{Anne Le Diberder}

1 Entre mémoire matérielle et immatérielle, la maison d'artiste est un lieu privilégié car fortement investi par l'aura de son occupant. C'est un univers patrimonial inspiré et parfois inspirant. Il y a dans la démarche de chaque visiteur une charge émotionnelle qui confère à ces lieux originaux une forme de sacralisation. S'ajoute le fait qu'une maison d'artiste est, par nature, un lieu intime où vie privée et espace de création s'imbriquent. Le visiteur y recherche l'étincelle de génie, tente de percevoir le processus créatif, plus que la découverte littérale ou linéaire d'une œuvre. Chaque objet, chaque pièce concourt à cette démarche. Le moindre objet devient relique et la maison son écrin protecteur, de sorte que contenant et contenu s'entremêlent, au point qu'il est quasiment impossible d'envisager l'un sans l'autre.

2 C'est particulièrement le cas pour la maison-atelier Foujita, dernière résidence du peintre franco-japonais Léonard Tsuguharu Foujita (1886-1968). Elle est située dans la vallée de Chevreuse, à Villiers-le-Bâcle, petit village du nord de l'Essonne, à une vingtaine de kilomètres au sud-ouest de Paris (fig. 1). 
Figure 1

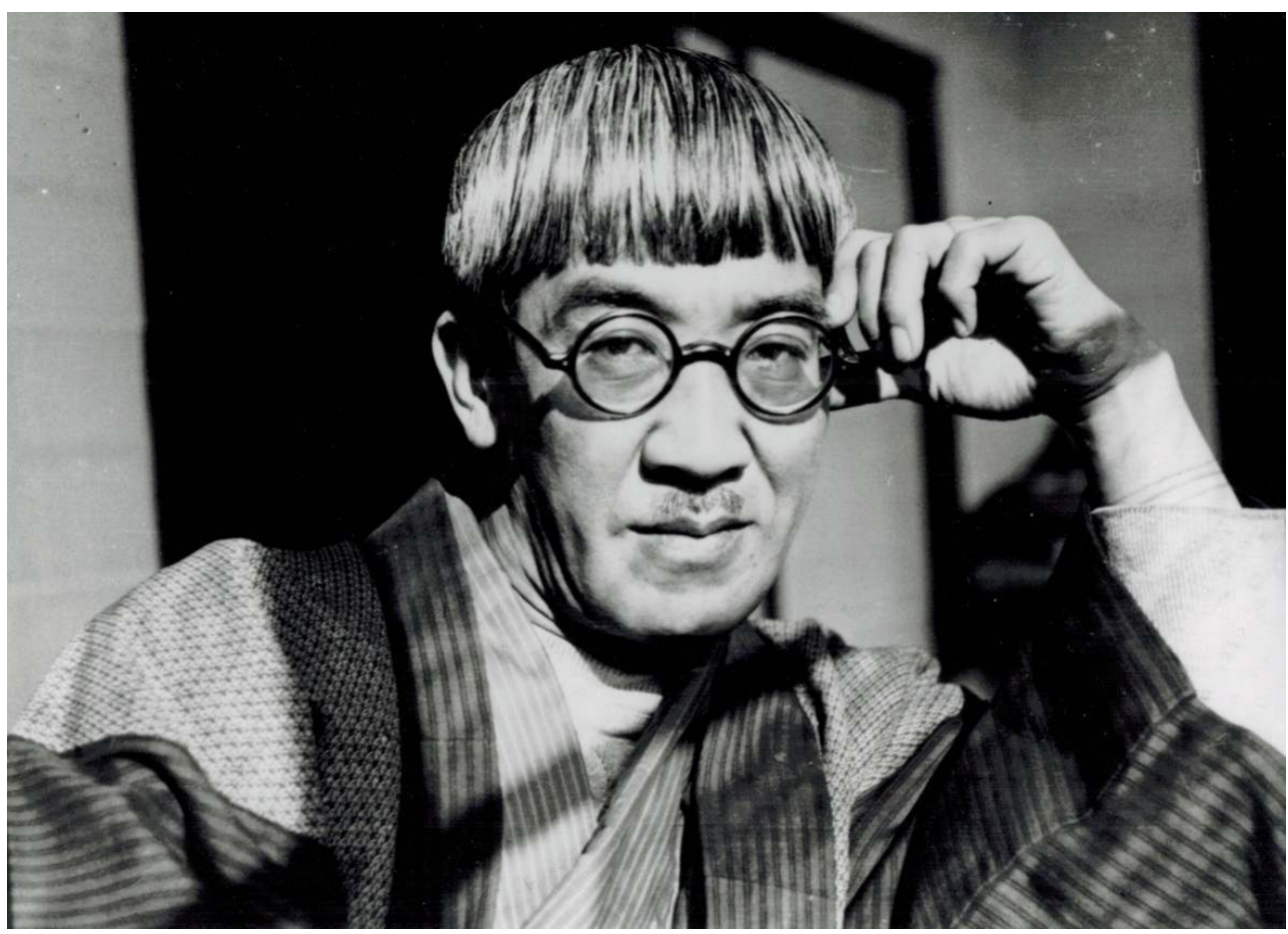

Portrait de Foujita.

Collection maison-atelier Foujita. (c) Conseil départemental de l'Essonne.

3 Léonard Foujita, l'un des peintres les plus en vue de l'entre-deux guerres, arrive à Paris en 1913. Il se lie d'amitié avec Modigliani, partage un atelier avec Soutine et s'intègre rapidement à l'univers bohème de Montparnasse. Après une première exposition remarquée en 1917, Foujita connaît la gloire dès le Salon d'automne de 1922. Il quitte Paris en 1930, voyage en Amérique latine et retourne au Japon qu'il quitte définitivement pour revenir s'installer en France en 1950.

4 Foujita retrouve alors Montparnasse et vit dans un appartement atelier situé 21 rue Campagne-Première. Mais en 1959, l'artiste se met en quête d'une maison à la campagne qui lui permettrait de travailler en toute sérénité. Il souhaite se rapprocher de Bièvres, où réside l'éditeur d'art Pierre de Tartas avec lequel il collabore, et du bourg voisin de Vauhallan, non loin de Saclay, où son ami le peintre japonais Yasse Tabuchi est installé.

5 C'est d'ailleurs ce dernier qui lui déniche cette petite maison rurale datant du XviII siècle, abritant deux anciens logis mitoyens destinés à des ouvriers agricoles. Quasiment en ruine, elle est bâtie sur un terrain en pente et arboré situé route de Gif, unique rue du village de Villiers-le-Bâcle. L'artiste en devient propriétaire en octobre 1960 et engage des travaux de rénovation importants qui dureront près d'une année (fig. 2). 
Figure 2

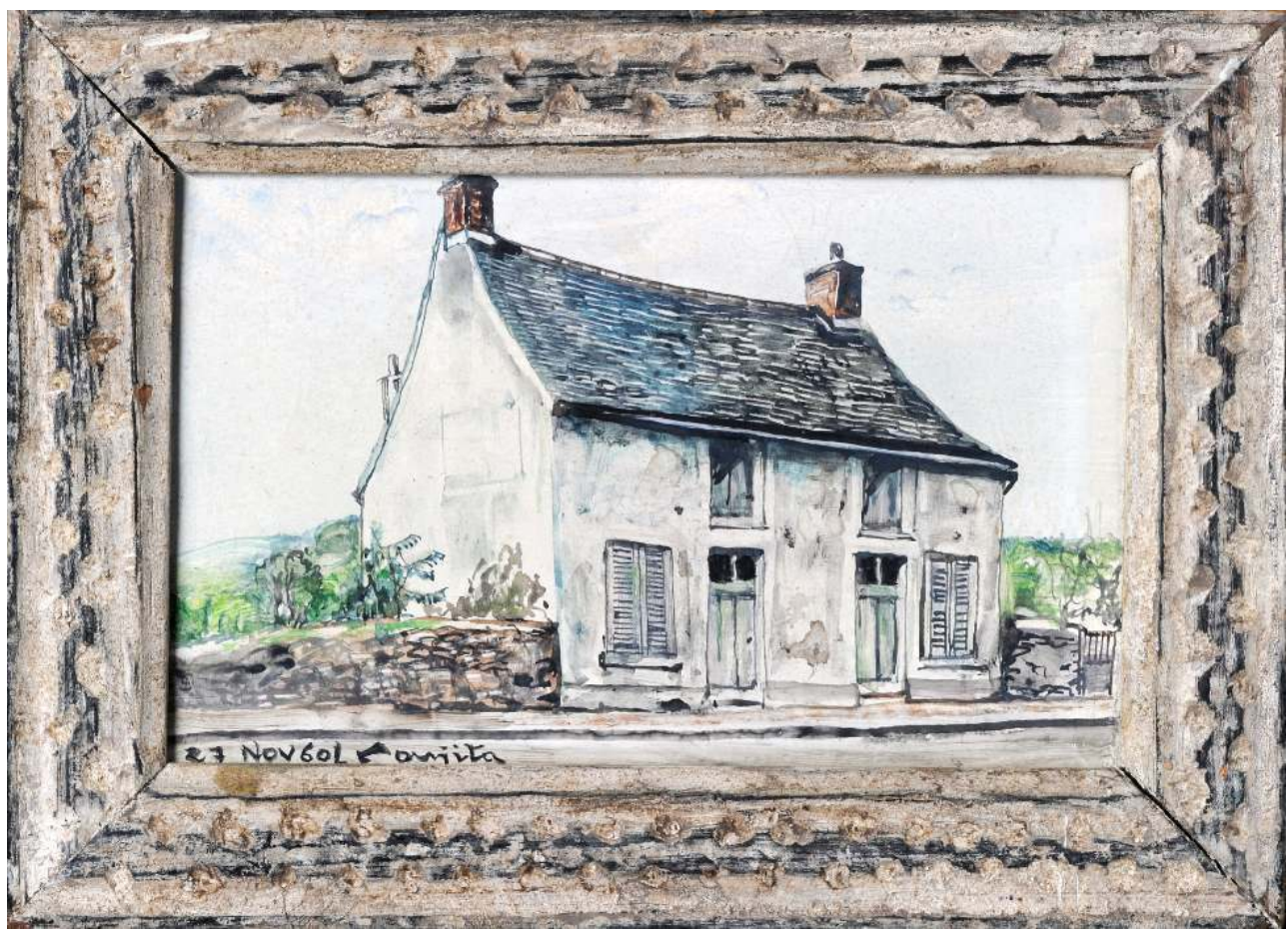

Notre maison. $12 \times 18 \mathrm{~cm}$, huile sur toile, 1960 .

Collection maison-atelier Foujita. (c) Fondation Foujita/ADAGP, 2016.

Foujita modifie en profondeur le bâti, éventrant la façade sud qui donne sur le jardin, aménageant chaque pièce en fonction de ses habitudes de vie. Il ne fait pas appel à un architecte mais s'entoure d'artisans installés dans les environs qui exécutent les travaux d'après ses dessins. Foujita suit le chantier avec attention, précis dans ses demandes jusque dans le moindre détail (fig. 3, fig. 4). 
Figure 3

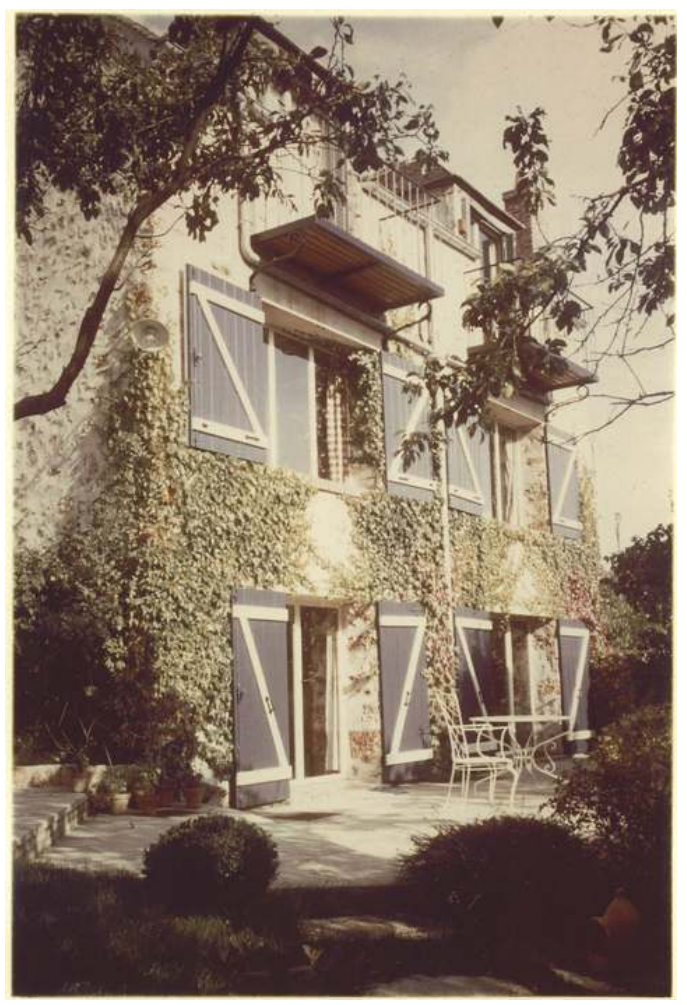

Façade sud après travaux.

(c) Conseil départemental de l'Essonne.

Figure 4

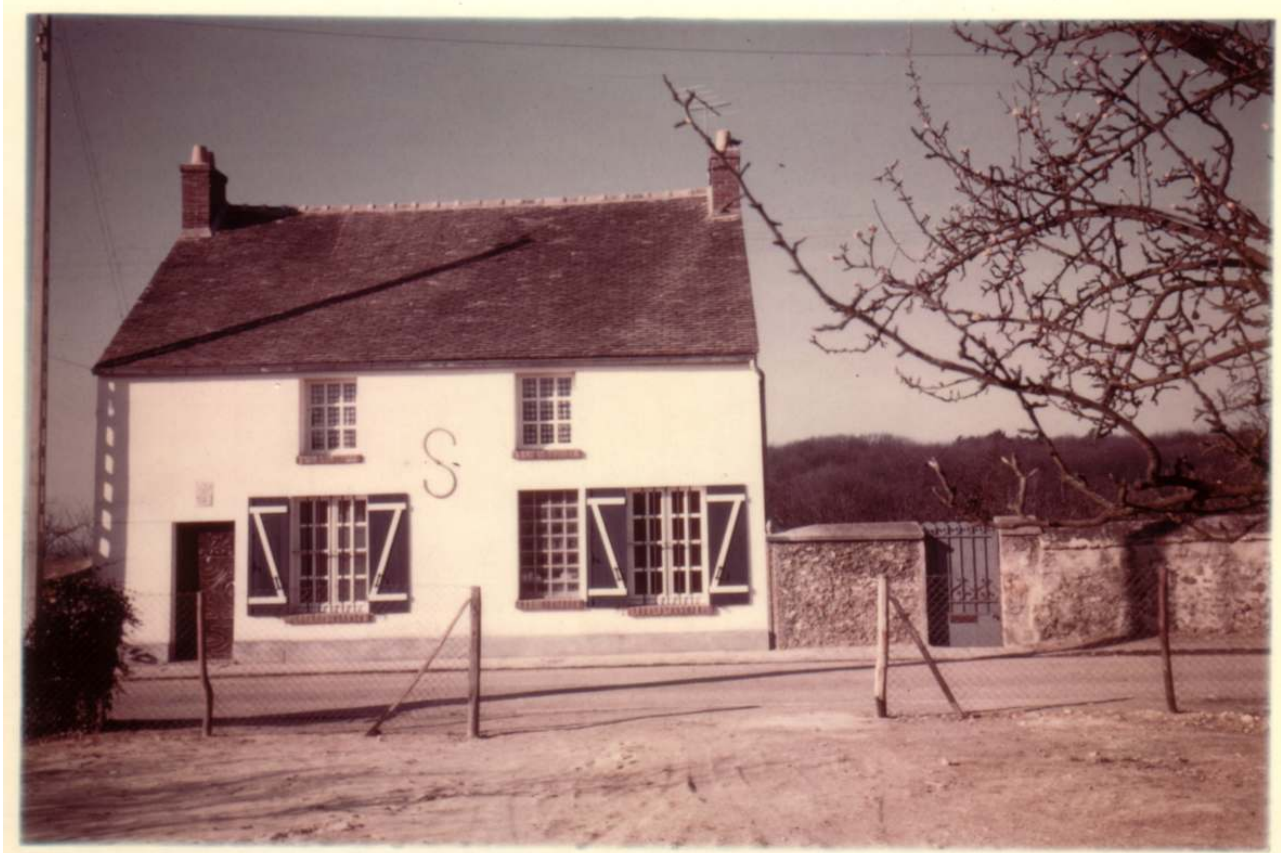

Façade nord après travaux.

(c) Conseil départemental de l'Essonne. 
7 Léonard Foujita lui-même avait envisagé que sa maison puisse être accessible au public après sa mort et exprimé le souhait que ses objets et collections encore en sa possession soient conservés in situ. Car il aimait pousser la porte des ateliers et s'imprégner de l'esprit des lieux, que ce soit celui de Cézanne à Aix-en-Provence, de Renoir à Cagnes-surmer ou de Gustave Moreau à Paris. Il avait le sentiment de mieux comprendre leur œuvre en découvrant leur intimité.

8 Paradoxe d'un homme qui veut offrir son atelier à la postérité, lui qui a toujours protégé jalousement son espace de création en brouillant les pistes. Il s'exposait en effet volontiers, jouant de son image de dandy, se prêtant au jeu de la célébrité, sans jamais rien révéler de son art et de ses techniques, refusant les visites impromptues dans son atelier.

9 C'est donc un vœu de son époux que Kimiyo Foujita exauce en faisant don de la maison et de son contenu au conseil départemental de l'Essonne en 1990, sous réserve que ce dernier l'ouvre au public.

10 L'enjeu était d'ouvrir cette petite maison tout en préservant l'authenticité des lieux. Cette démarche imposait de prendre en considération non seulement les collections, le bâtiment qui l'abritait, mais aussi les attentes du public. Il convenait donc d'étudier avec minutie l'articulation entre ces trois éléments.

11 En effet, les exigences, tant de conservation que d'accueil du public, peuvent se heurter à des contraintes liées à la nature de ces maisons d'artiste. La responsabilité des collections impose des précautions de conservation spécifiques, car l'objectif majeur est d'en assurer au mieux la pérennité. Dans un lieu de mémoire, ces objets font partie d'un ensemble décoratif choisi par son illustre propriétaire, l'environnement doit impérativement être pris en compte. Ici, tout est affaire de compromis, d'équilibre entre les exigences de sécurité et de conservation et le sens voulu par l'artiste dans ses choix d'accrochage ou de décor. En outre, il faut composer avec le bâti. Il est exceptionnel qu'une maison réponde aux exigences d'un établissement recevant du public. Là encore, une démarche pragmatique s'impose, elle s'accompagne de décisions importantes, car souvent irréversibles. Ouvrir une maison d'artiste suppose donc de s'interroger sur ces enjeux et nécessite une réflexion approfondie préalable à tout choix scénographique, a fortiori à un réaménagement de l'espace.

12 C'est la démarche qui a été adoptée pour la maison-atelier Foujita. Elle repose sur une connaissance approfondie des lieux et l'élaboration de documents qui ont servi à la fois d'aide à la décision et de base documentaire.

\section{Un état des lieux exhaustif}

13 La donation effectuée par Kimiyo Foujita en faveur du conseil départemental de l'Essonne en 1990 comprenait à la fois un patrimoine immobilier, maison et terrain, mais aussi le mobilier. Il importait de rendre compte précisément de l'un comme de l'autre.

\section{Une prise de connaissance du bâti}

14 Le caractère radical de la réhabilitation menée par Foujita est peu visible depuis la rue qui a conservé son aspect d'origine, et cette petite maison d'un seul étage passe d'ailleurs totalement inaperçue dans le village tant son aspect est anodin. Rien ne signale 
l'existence d'un lieu remarquable ici. En revanche, côté jardin, la bâtisse d'origine, presque aveugle, laisse désormais place à une façade largement ouverte, où se devinent les trois étages de la maison.

Les modifications intérieures sont plus évidentes: Foujita a su tirer parti de tous les espaces, créant une circulation astucieuse entre les pièces et même les usages de celles-ci. Des détails démontrent l'attention extrême que Foujita a portée à son intérieur. On peut noter aussi des partis inhabituels : ainsi, il a opté pour des fenêtres à petits carreaux côté rue, mais pour de grandes fenêtres vitrées côté jardin, rompant avec les principes d'homogénéité de style des ouvrants qui prédomine généralement dans une maison. Entre l'entrée et le salon, Foujita a choisi d'animer le mur de séparation par une fenêtre intérieure ornée d'un décor en fer forgé réalisé par un artisan d'après les dessins de l'artiste. Il a fait également réserver des niches dans certains murs, ceux de la salle à manger et de la chambre.

Tous ces éléments renvoient à la vie de l'artiste. Grand voyageur, plus attaché à ses objets qu'aux villes ou pays qu'il a traversés, il a toujours tenté de traduire ses rencontres et ses chocs esthétiques à travers ses intérieurs successifs. D'où l'inspiration hispanique de la fenêtre intérieure, et les influences extrême-orientales, métissées à l'esprit occidental, décelables au fil des pièces (fig. 5, 6, 7).

Figure 5

Maison Foujita _ Sous. sol Elévation intérieure des murs de la salle à manger Echelle 1/20

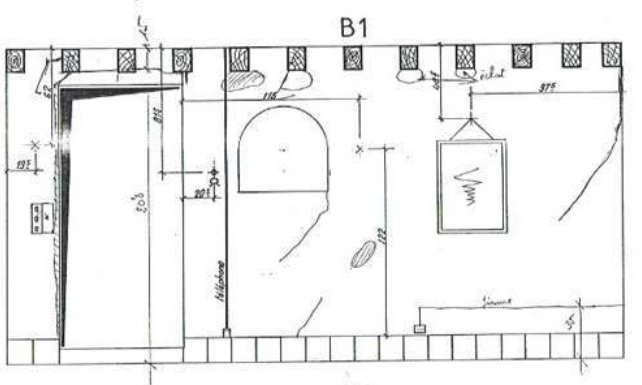

B3

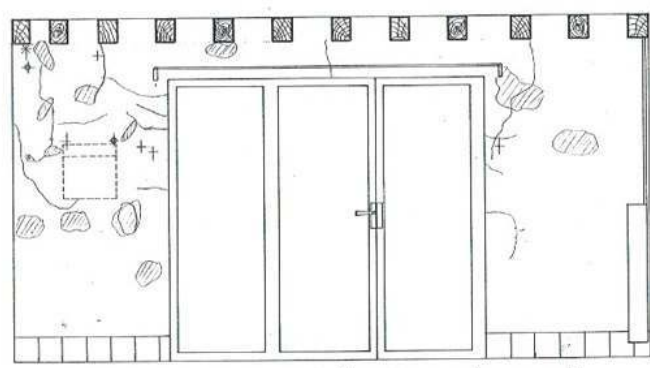

Légende: $*$ cheville \& trou + clou $x$ erochet

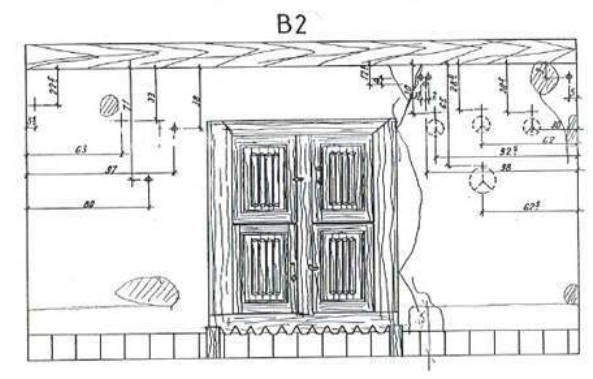

B 4

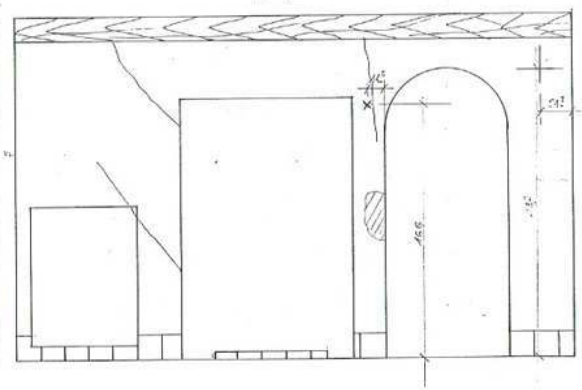

Relevé du bâti : Sous-sol. Élévation intérieure des murs de la salle à manger.

(C) Conseil départemental de l'Essonne. 
Figure 6

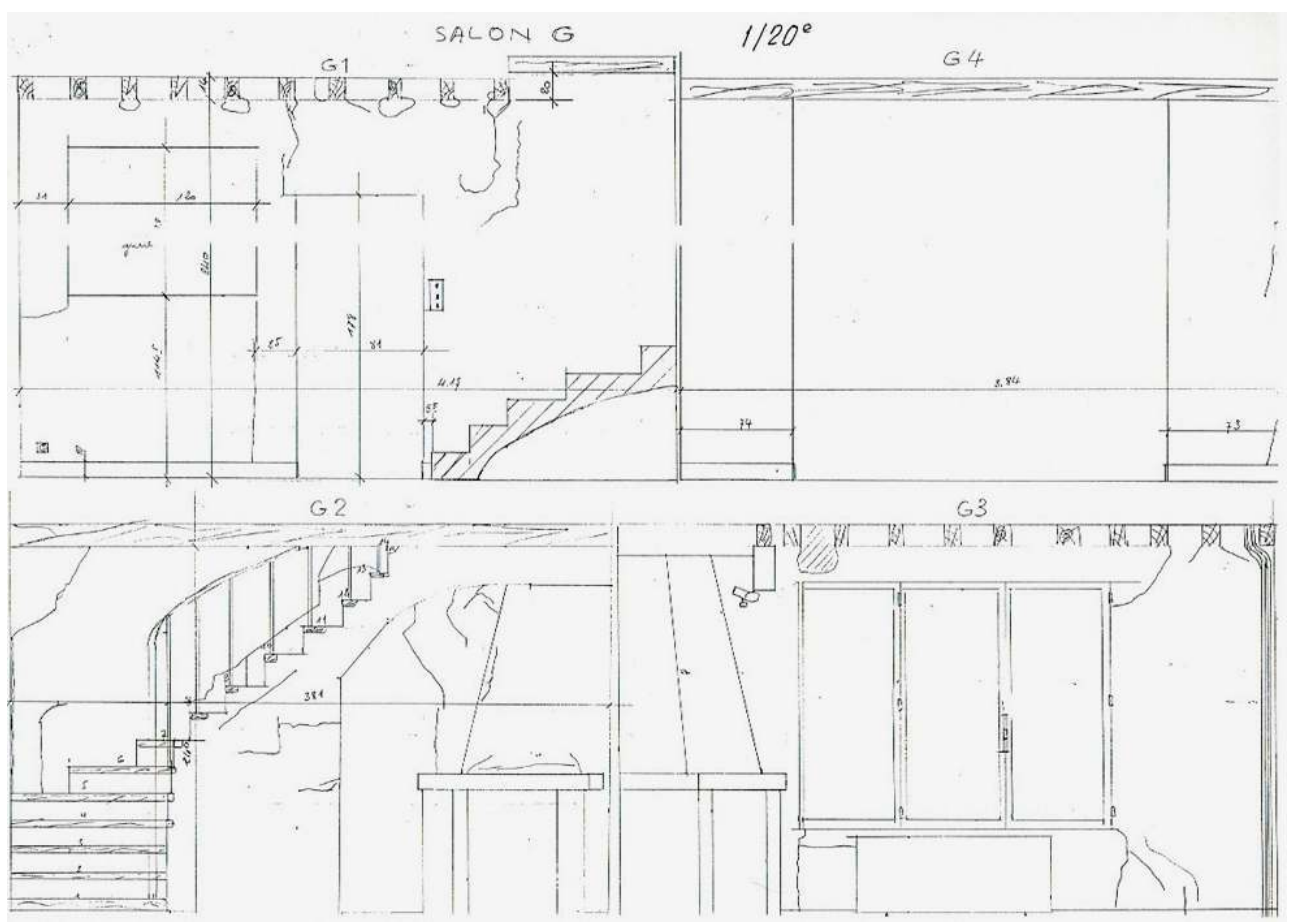

Relevé du bâti : Salon

(c) Conseil départemental de l'Essonne.

Figure 7

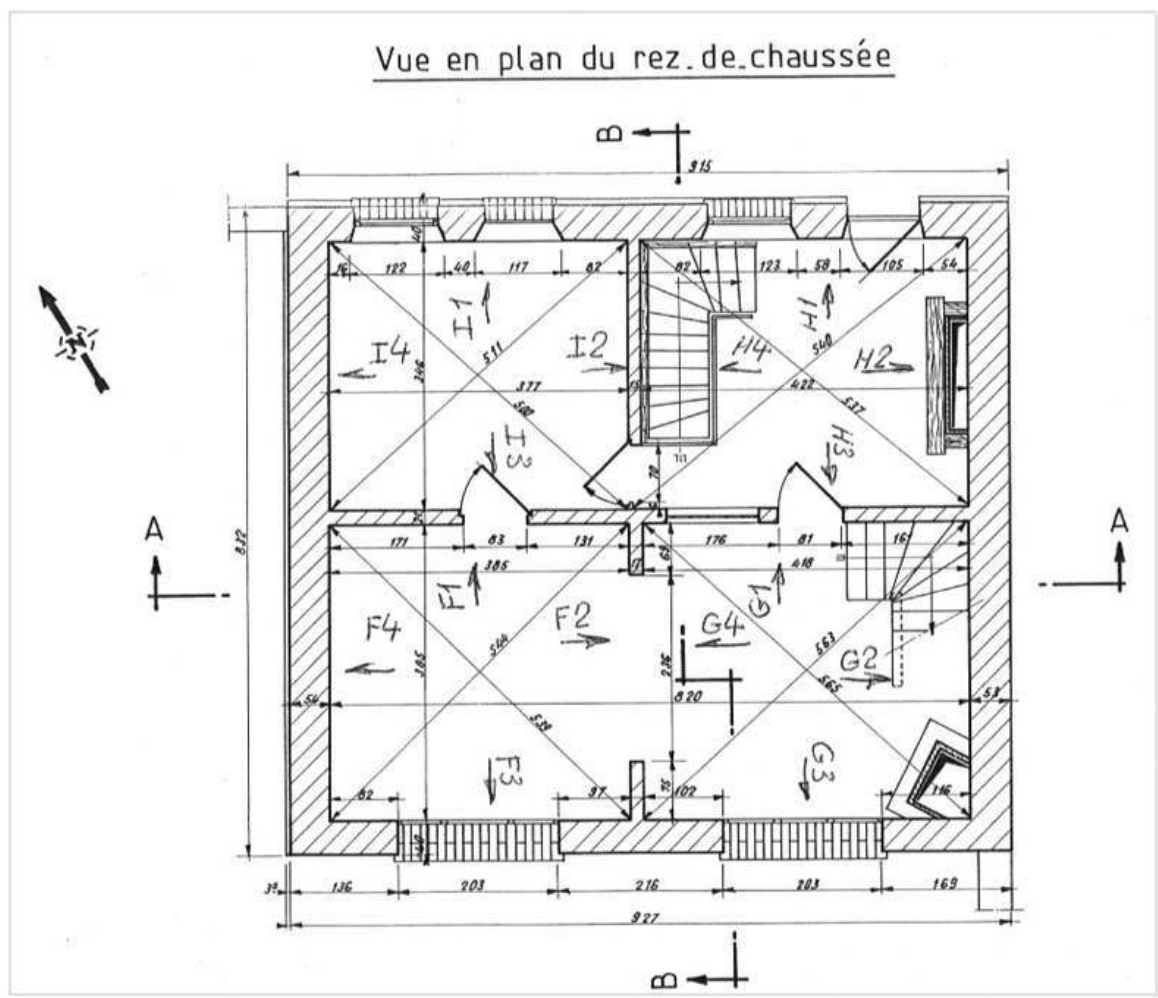

Relevé du bâti : Vue en plan du rez-de-chaussée.

(c) Conseil départemental de l'Essonne. 
17 Au dernier étage de la maison, Foujita a aménagé les combles en atelier. Il va à l'encontre des usages et de son confort de peintre en faisant ouvrir plein sud deux portes-fenêtres qui donnent sur deux petits balcons. C'est dans cet atelier que le peintre réalisa ses derniers grands projets. Il y conçut notamment la chapelle Notre-Dame-de-la-Paix de Reims entre 1964 et 1966, date de la consécration de l'édifice. L'artiste s'est d'ailleurs servi du pignon ouest pour réaliser un vaste essai de peinture murale. C'est l'existence de cette peinture murale qui a justifié l'inscription de la maison au titre des monuments historiques en 1994, avant même son ouverture au public ${ }^{1}$.

18 Afin de conserver la mémoire du site avant tous travaux, des relevés complets du bâti ont été effectués, ils comprennent également les traces d'usage visibles tels les fissures, les marques d'usure, les clous. Le dossier est enrichi de clichés pris par l'artiste pendant l'année de travaux, de ses plans et dessins. Cette documentation a permis d'identifier avec précision les étapes de la réhabilitation voulue par l'artiste et de constituer des archives précieuses pour l'histoire du lieu (fig. 8).

Figure 8

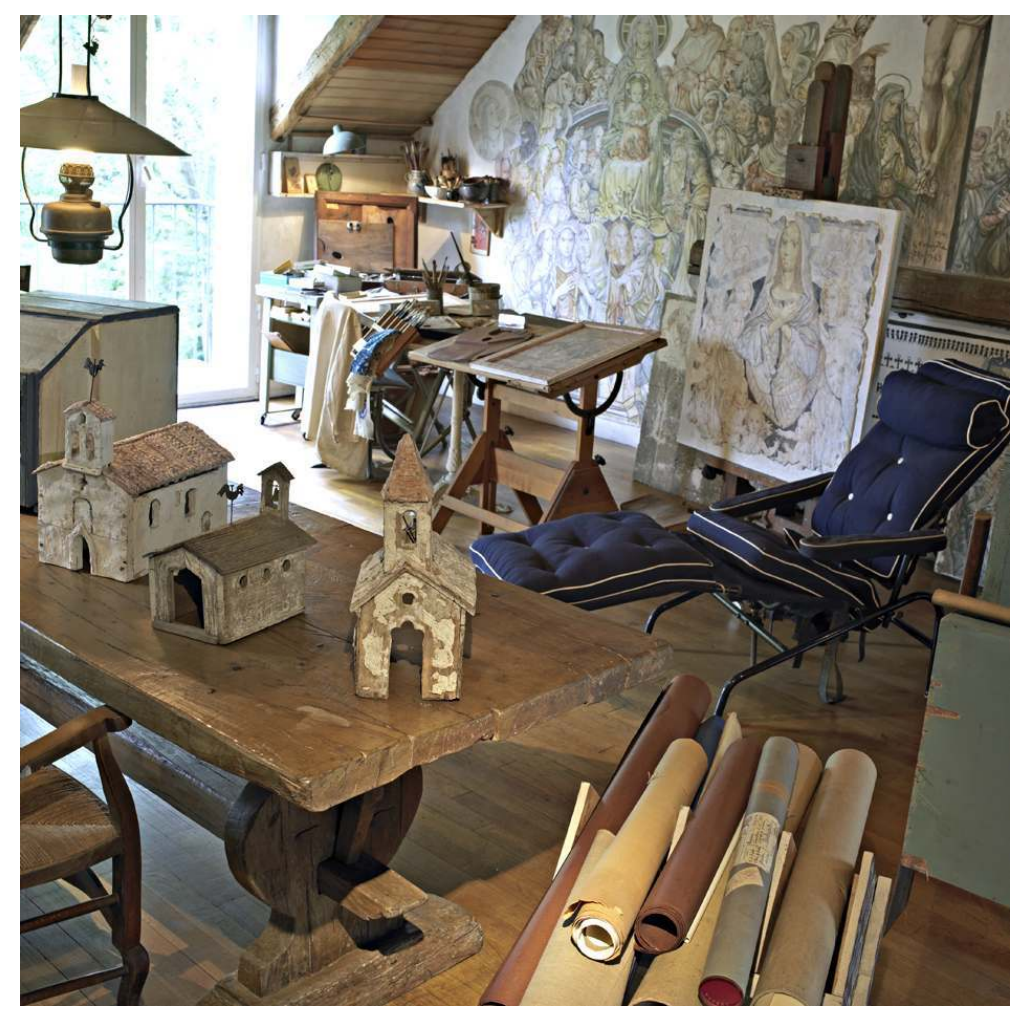

Vue de l'atelier de peinture murale.

Phot. Godard, Laurence. (C) CD91/Fondation Foujita/ADAGP, 2016.

\section{L'inventaire du mobilier}

19 La donation comprenant le mobilier resté sur place, une campagne de récolement a été entreprise. Plus de 3000 objets ont été répertoriés, allant du matériel d'atelier, des œuvres ou esquisses jusqu'aux menus objets comme les ustensiles de cuisine. Leur emplacement d'origine a été soigneusement noté en vue d'un déménagement avant l'engagement de travaux sur le bâti. 
20 et inventaire a révélé une facette méconnue de l'artiste. Celui-ci était particulièrement sensible à toutes les formes d'artisanat, il fréquentait les marchés aux puces et achetait verreries, céramiques et autres objets parfois insolites comme des pesons et fléaux. Il était également bricoleur et n'hésitait pas à façonner de petits objets usuels comme des arrosoirs à partir de boîtes de conserves. Plus surprenant encore, Foujita, qui avait appris à tisser et à coudre, réalisait lui-même ses rideaux ou ses coussins (fig. 9, fig. 10).

Figure 9

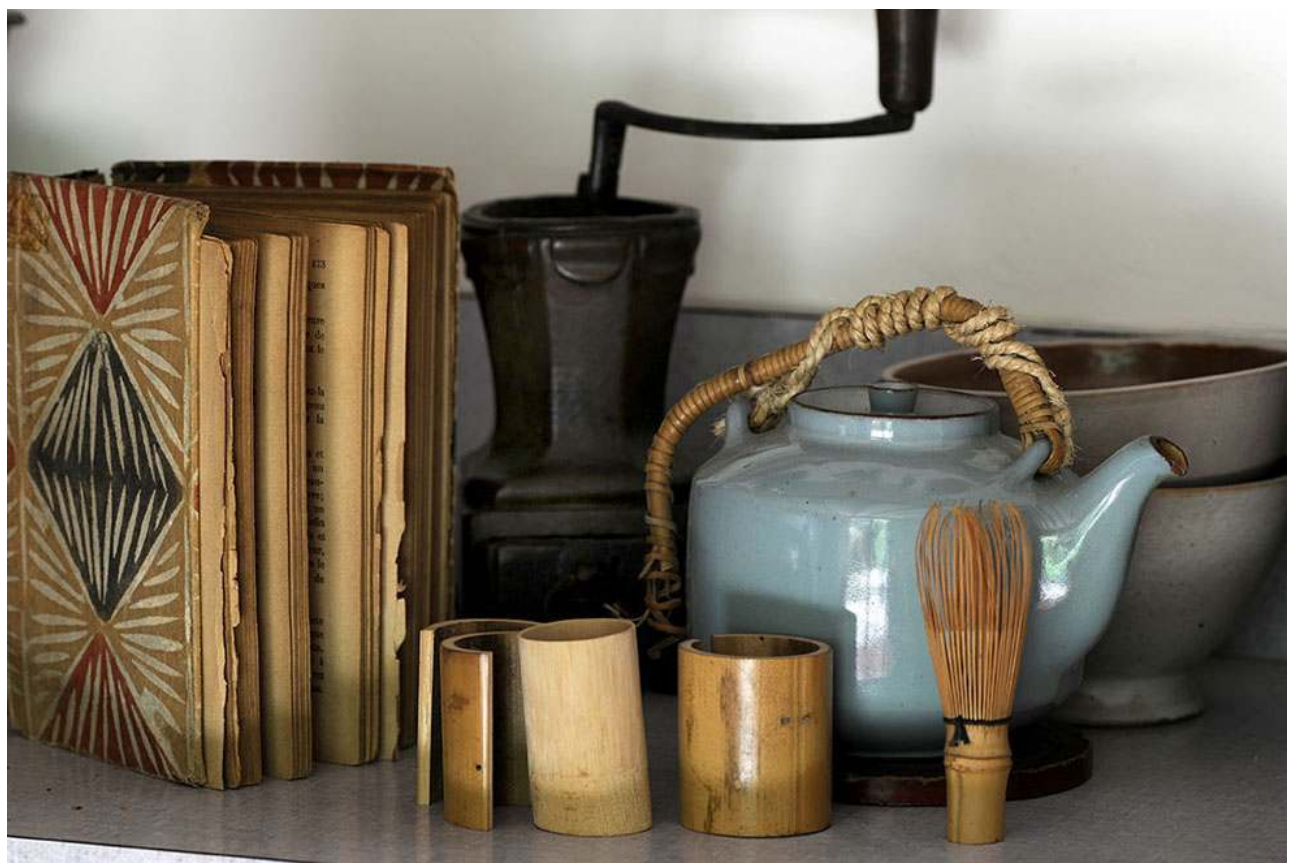

Objets mobiliers de la maison Foujita.

Phot. Godard, Laurence. (C) Conseil départemental de l'Essonne.

Figure 10

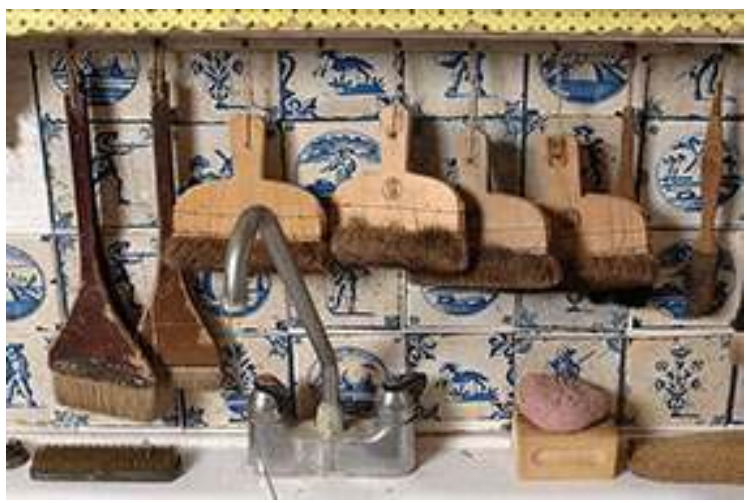

Objets mobiliers de la maison Foujita.

Phot. Godard, Laurence. (C) Conseil départemental de l’Essonne.

L'étude de ces objets nous permet de mieux cerner l'univers très éclectique de Foujita, autant attiré par l'art populaire, le mobilier ancien, souvent acquis auprès d'antiquaires, que par le design. Son salon est ainsi meublé d'une table basse dessinée par l'architecte 
danois Finn Juhl (1912-1989) et d'un canapé conçu par l'Italien Marco Zanuso (1912-2001) pour l'éditeur Artflex (fig. 11).

Figure 11

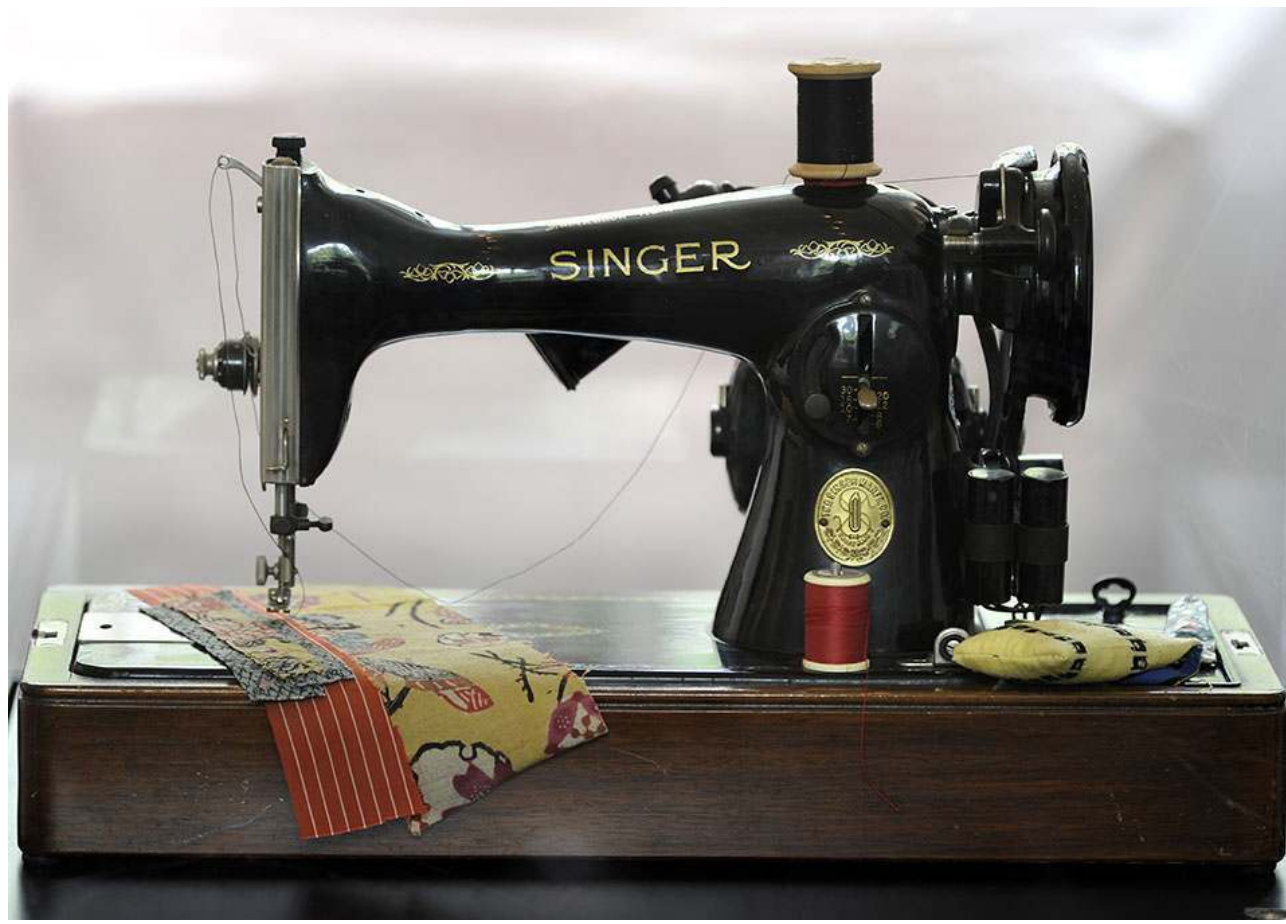

Objets mobiliers de la maison Foujita.

Phot. Godard, Laurence. (C) Conseil départemental de l'Essonne.

L'importance que l'artiste confère à l'aménagement de son intérieur est donc tout à fait singulière. Tout a été ordonnancé avec minutie, ce qui justifie que l'inventaire des objets n'ait pas été dissocié du travail sur le bâti. En parallèle, une recherche documentaire a permis l'acquisition d'un précieux reportage photographique sur cette maison. Celui-ci a été réalisé en 1965 par Paul Almasy (1906-2003) dans le cadre d'un reportage publié dans le mensuel Jardin des Arts en octobre $1965^{2}$. Enfin, le recueil de la mémoire de la veuve de l'artiste a aidé à affiner la localisation des objets dans l'atelier, certains ayant été mis dans des cartons après le décès du peintre. Tout ce travail préalable a contribué à la bonne connaissance du site et des collections. Il a constitué la phase conservatoire qui s'est achevée par le déménagement de l'ensemble des collections dûment répertoriées, leur emplacement dans la maison noté sur l'emballage de chacun d'entre eux. Ce travail préalable a été déterminant dans le parti scénographique retenu et les décisions portant sur le bâti en ont découlé (fig. 12, fig. 13). 


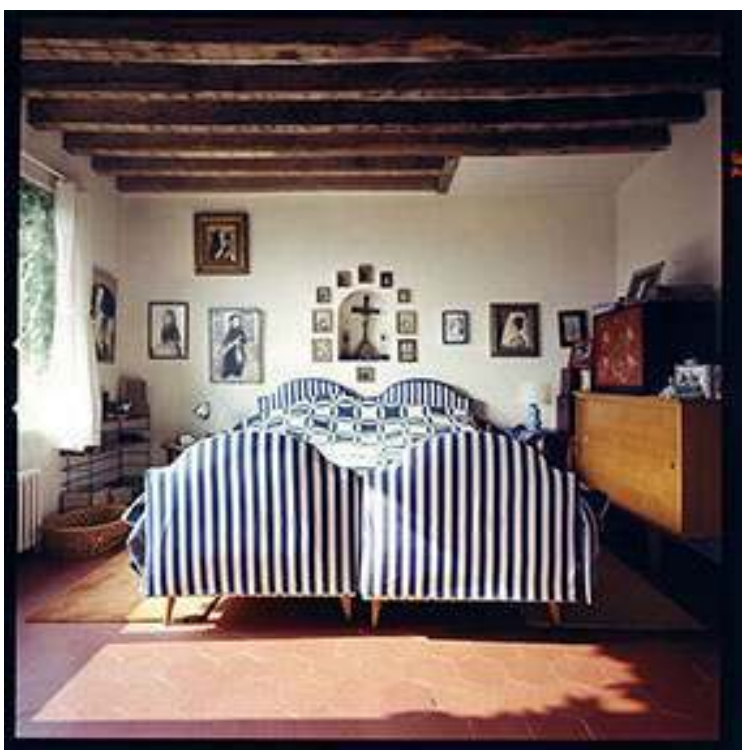

Vue de la maison du peintre Foujita.

Phot. Almassy, Paul, 1965. @ Conseil départemental de l'Essonne.

Figure 13

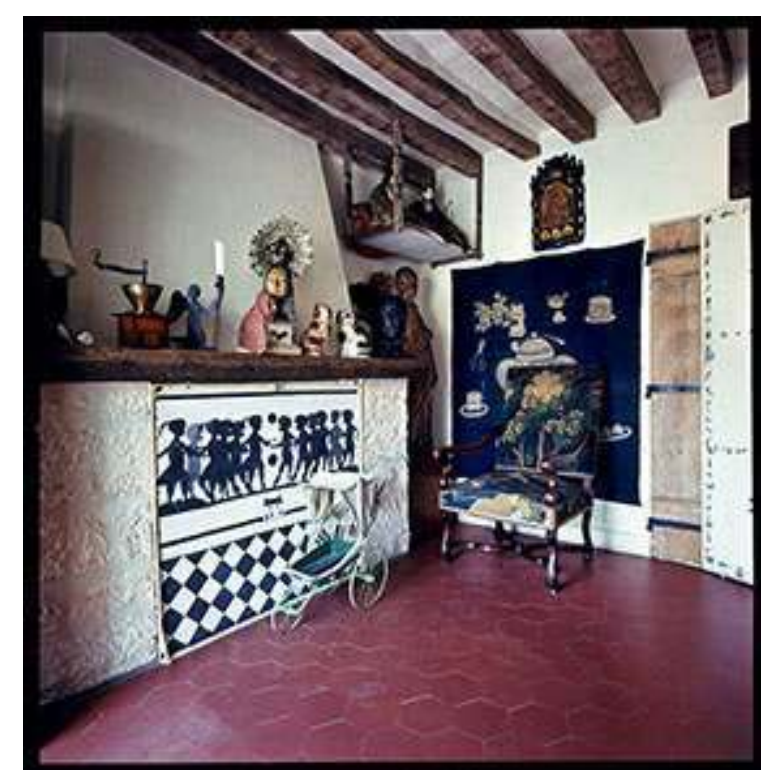

Vue de la maison du peintre Foujita.

Phot. Almassy, Paul, 1965. (C) Conseil départemental de l'Essonne.

\section{Le choix muséographique}

Le parti choisi n'a pas dissocié objets et maison, tant leur lien organique était évident, même si leur niveau de protection diffère quelque peu, puisque la maison est inscrite, tandis que les collections sont pour partie classées; cette protection s'applique aux œuvres et objets d'art les plus importants réalisés par Foujita - soit 158 œuvres -, 
525 autres objets sont inscrits, les 1818 autres ne bénéficient d'aucune protection. En dépit de ce traitement juridique hiérarchisé, la phase d'analyse a mis en évidence la nécessité d'avoir une approche globale du lieu, la dissociation du bâti et des collections pouvant entraîner une perte de cohésion par la prévalence d'un point de vue sur l'autre. Cependant, un impératif commun a été mis en avant : l'obligation de réversibilité de toute intervention. Bâti et collections ayant été fortement investis par Foujita, nous sommes face à une maison-relique, laissant peu de place à la réinterprétation du lieu. Quelle que soit sa valeur patrimoniale, chaque objet a sa place dans cette maison et donne un sens à celle-ci.

Foujita, nous l'avons vu, a mis en scène son quotidien. Cependant, il ne le théâtralise pas, bien au contraire, il accumule modestement objets et souvenirs. L'ensemble concourt à créer un univers intime dans le prolongement de son travail artistique. Il importait de conserver cette modestie qui impose une proximité avec l'objet. En outre, dès que l'on observe cette collection, une familiarité s'instaure avec l'univers du peintre car il a souvent représenté ses objets du quotidien dans ses tableaux. Ils servent alors de décor aux portraits, ou s'insèrent dans les natures mortes : nous en retrouvons certains dans sa maison de Villiers-le-Bâcle. Cette proximité est renforcée par le fait que Foujita annote chaque pièce qu'il considère importante. Il y indique la date d'acquisition, parfois le lieu ou les circonstances de l'achat.

5 La solution d'une remise en place de tous les objets inventoriés et présents sur les documents photographiques de 1965 s'est rapidement imposée. En revanche, ont été mis en réserve tous les objets postérieurs à 1968, date de la mort du peintre.

Nombre de questions ont surgi à propos de la nécessité ou non d'imposer des mises à distance en raison de la quantité d'objets et de l'étroitesse des lieux. C'est justement cette spécificité qui a permis d'orienter la décision. En effet, il semblait déraisonnable d'ouvrir cette maison sans accompagnement. Le principe de visites exclusivement guidées avec un nombre restreint de visiteurs (15 au maximum) acté, la décision de ne pas installer de mise à distance a été prise, afin de conserver au plus juste l'atmosphère voulue par l'artiste.

\section{Les interventions sur le bâti}

7 Nous l'avons déjà souligné, bâti et collections sont, dans ce lieu de mémoire, étroitement liés. Aussi, aucune modification du bâti n'a été entreprise, à l'exception de la surélévation de quelques centimètres de la toiture, pour permettre le passage de fourreaux électriques et éviter ainsi toute goulotte ou tout ajout visible dans l'atelier sous combles (fig. 14, 15, 16). 
Figure 14

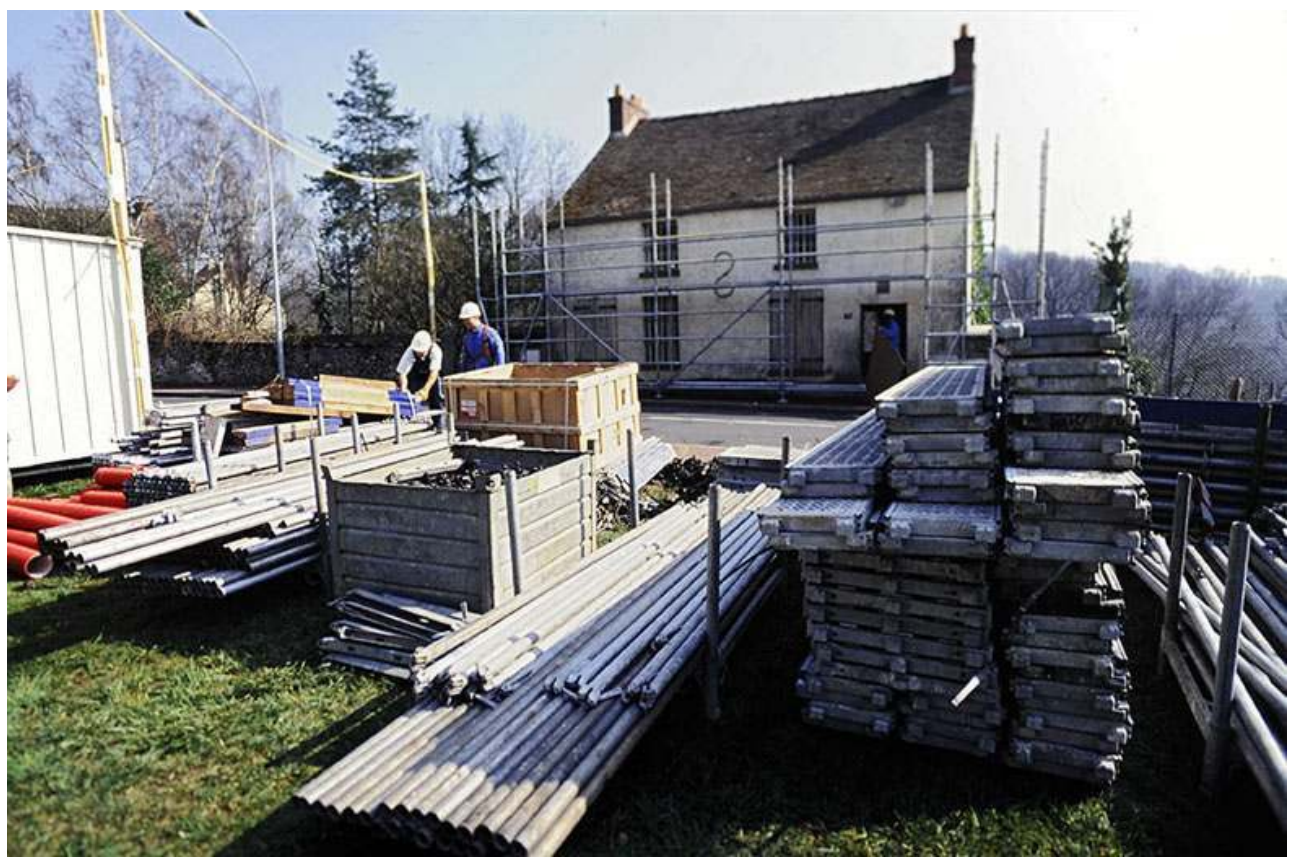

Surélévation de la toiture.

Phot. Godard, Laurence. (C) Conseil départemental de l'Essonne.

Figure 15

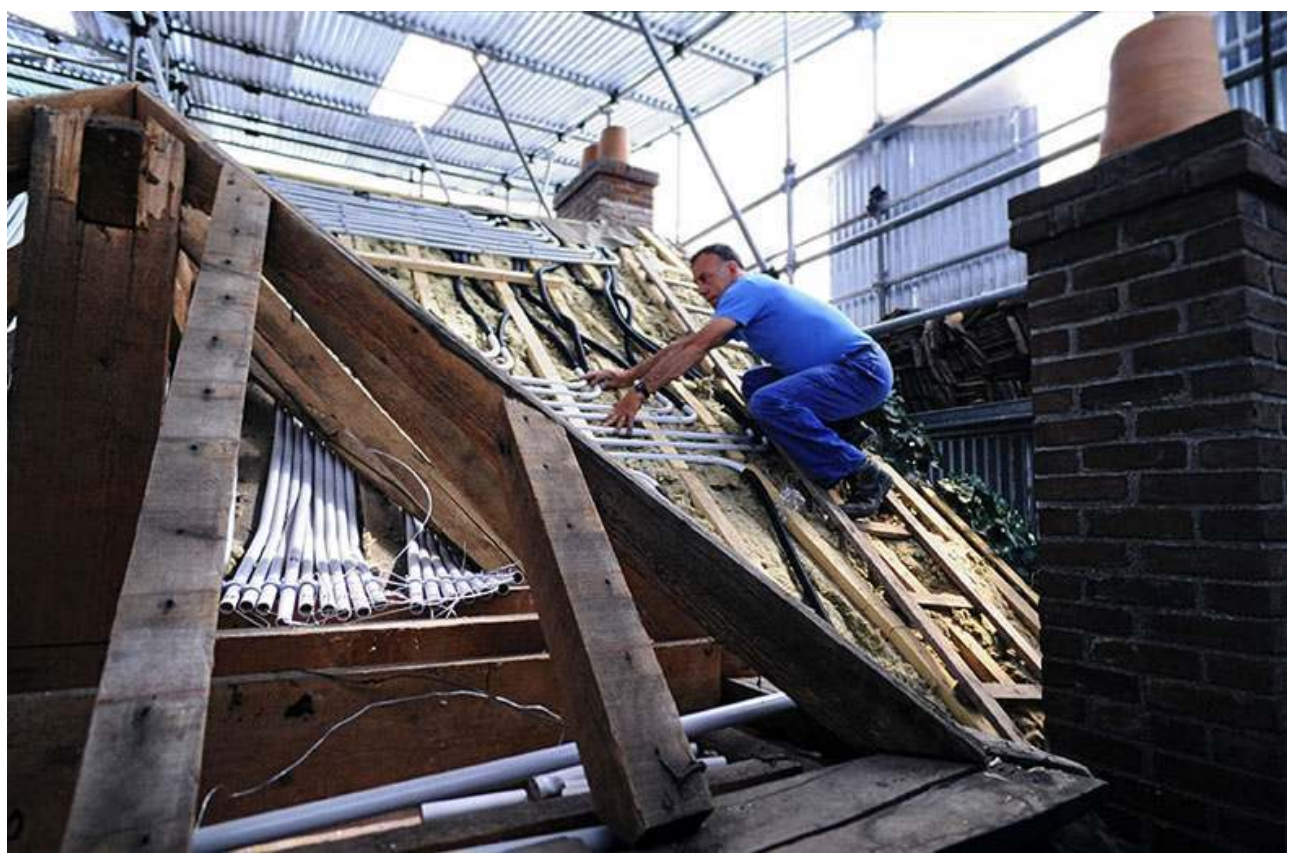

Surélévation de la toiture.

Phot. Godard, Laurence. (c) Conseil départemental de l'Essonne. 
Figure 16

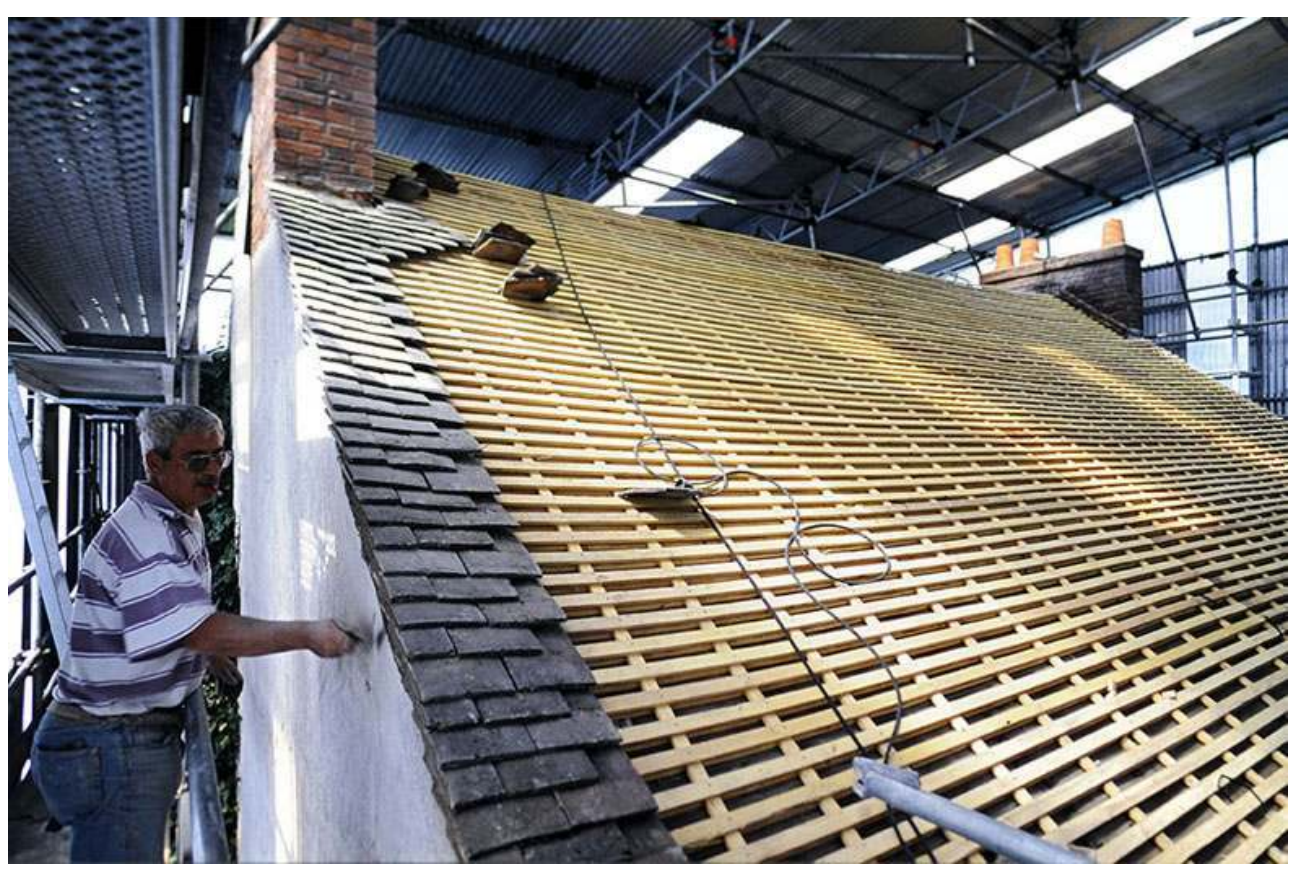

Surélévation de la toiture.

Phot. Godard, Laurence. (C) Conseil départemental de l'Essonne.

28 Le choix de Foujita d'une peinture blanche uniforme dans l'ensemble des pièces a facilité leur restauration, qui aurait peut-être été plus complexe si nous avions été en face de tapisserie ou de papier peint. Les murs de l'atelier, qui n'avait pas été touché depuis 1968, ont été nettoyés à la gomme par une équipe de restaurateurs. Les deux autres étages, occupés très régulièrement par Kimiyo Foujita jusqu'en 1990, ont été repeints en blanc avec une peinture à la colle similaire à l'originale. Les ouvrants ont été conservés, les vitres remplacées par des verres anti-UV et anti-effraction. Les volets ont été doublés par un blindage et le système d'alarme, déjà existant, a été modernisé et renforcé. Tous les éclairages d'origine ont été conservés mais remis aux normes, sans que leur aspect soit modifié.

29 Afin de préserver au mieux l'atelier, pièce majeure du site, les fenêtres donnant plein sud ont été occultées par des stores intérieurs. L'éclairage est assuré par les lampes d'origine, complété par des points lumineux permettant de scénographier uniquement par un jeu de variation lumineuse un atelier riche en objets très divers. Foujita $\mathrm{y}$ travaillait près de 10 heures par jour et avait aménagé l'espace de façon rationnelle. La mise en place d'une programmation lumineuse - équipée de LED depuis 2014 - activée par une télécommande permet de séquencer la visite en fonction de ces zones d'activités voulues par le peintre, et de développer une médiation culturelle adaptée aux attentes du groupe de visiteurs (zoom sur les techniques, le mode de vie, etc.).

30 La proximité avec les objets étant préservée, le sentiment d'être dans un lieu vivant, encore habité, participe fortement au succès du site. La médiation culturelle aide également les visiteurs à percevoir la personnalité de l'artiste. L'atelier impressionne toujours en raison de son exceptionnel état de conservation. 


\section{Les limites d'une « maison-relique »} documentation d'archives: correspondances, photographies, etc. afin de constituer un fonds biographique de référence. Ce choix n'exclut pas l'acquisition d'œuvres correspondant à la période d'installation à Villiers-le-Bâcle. C'est ainsi que le conseil départemental s'est récemment porté acquéreur d'une eau-forte sur plaque de cuivre figurant les cavaliers de l'Apocalypse destinée à l'illustration du célèbre livre L'Apocalypse édité par Joseph Forêt ${ }^{3}$. L'objectif de toute acquisition est de compléter la connaissance des techniques utilisées par Foujita ou de contribuer à enrichir le fonds biographique (fig. 17). 
Figure 17

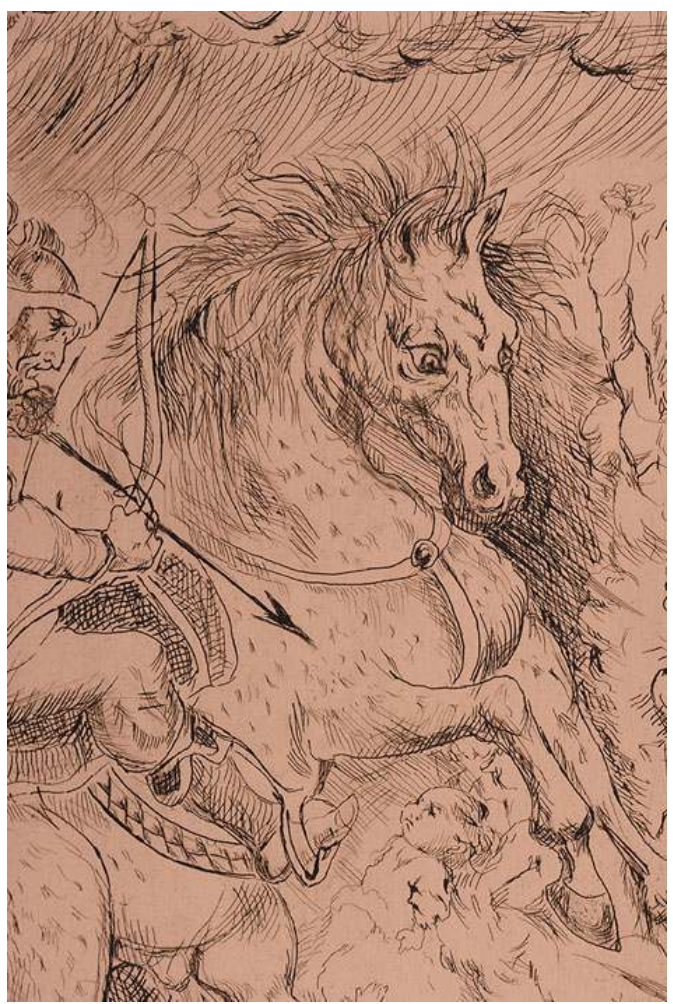

Apocalypse, détail, eau-forte sur plaque de cuivre, 1960.

Phot. Godard, Laurence. (C) CD91/Fondation Foujita/ADAGP 2016.

La contrainte majeure de ce lieu de mémoire est de parvenir à trouver un équilibre entre le besoin de reconnaissance publique, en particulier par un flux de visiteurs annuel qui justifie l'investissement de fonds publics, et la protection de ce patrimoine, difficilement compatible avec l'accueil d'un public en très grand nombre.

\section{Les enjeux des années à venir}

Depuis son ouverture au public, en 2000, la maison-atelier Foujita connaît une hausse régulière de sa fréquentation. Elle a dépassé en 2014 les 6500 visiteurs. Nous sommes à un seuil qui requiert une nouvelle réflexion approfondie. Celle-ci porte sur un compromis à trouver entre le désir d'accroître la jauge d'accueil, en raison du succès de l'établissement, et la fragilité du lieu.

Afin de préserver l'intégrité de la maison et d'organiser l'accueil du public, le conseil départemental a opté pour l'acquisition de la propriété voisine, un ancien presbytère datant du début du xx siècle. Cela permet, d'une part, de constituer un écrin autour de la maison du peintre grâce à l'existence d'un jardin de $2000 \mathrm{~m}^{2}$, d'autre part, d'organiser un espace d'accueil et de préparation à la visite. Cette bipolarité préserve la maison, conforte son statut de maison-relique et favorise la gestion du flux de visiteurs si nécessaire.

La maison et son jardin constituent un ensemble apprécié pour son charme et accroître démesurément son ouverture pourrait détruire cette harmonie. L'objectif principal est de maintenir au mieux les conditions de conservation actuelles en optimisant l'accueil et en 
le rendant plus confortable, en particulier lors de l'accueil de groupes. La jauge de la maison restant fixée à 15 visiteurs, il importe de développer les propositions pour que l'attente des visiteurs se déroule dans les meilleures conditions.

41 La fragilité de la maison, construite sur un terrain en pente, et dont la façade sud, fortement modifiée, affaiblit encore la structure non chaînée, impose un suivi précis, mené par le conseil départemental sous le contrôle de la conservatrice des Monuments historiques de la DRAC d'île-de-France et de l'architecte des Bâtiments de France, territorialement compétents. Cette fragilité, l'agrément du lieu requièrent de toute évidence une ouverture raisonnée et raisonnable du lieu. La jauge maximale est estimée entre 10000 et 15000 visiteurs annuels. Au-delà de ce nombre, une trop grande fréquentation des lieux pourrait avoir de lourdes incidences sur le bâti, inadapté à un flux de visiteurs dense et permanent pour les raisons exposées précédemment. Il faudrait également revoir la présentation des collections afin de les protéger plus drastiquement des modifications de climat et des risques de dégradation engendrées par un public très important. Il en résulterait une modification profonde du site qui lui ferait perdre une grande partie de son sens.

en compte ces paramètres et visent à concevoir un nouve aménagement des espaces dévolus à l'accueil afin de proposer une lecture complémentaire à la visite de la maison-atelier et d'en renforcer le discours. Celui-ci s'appuie sur trois axes : expliquer la trajectoire exceptionnelle d'un artiste japonais qui a choisi de vivre en France sans jamais renier ni ses racines ni son pays d'adoption, faire découvrir un atelier d'artiste préservé. Enfin, mettre en valeur les techniques artistiques qui permettent de faire comprendre, au jeune public en particulier, que derrière une œuvre ou un objet d'art, il y a une main, une volonté, un savoir-faire.

Toutes ces actions culturelles ont pour objectif de mieux faire découvrir et aimer un ensemble mobilier singulier qui prend toute sa valeur et son unicité à travers l'histoire d'un homme et d'une maison.

\section{BIBLIOGRAPHIE}

JOLY, Marie-Hélène. Musées biographiques, maisons-musées, musées littéraires. Dossier documentaire pour le centre de documentation de l'École nationale du patrimoine. Paris : 1997.

GRIBENSKI, Jean, MEYER, Véronique, VERNOIS, Solange (dir). La maison de l'artiste : construction d'un espace de représentations entre réalité et imaginaire (XVII $-X X^{e}$ siècles). Rennes : Presses Universitaires de Rennes, 2007.

SCIPION, Sylvie-Marie. « Ouvrir les portes d'une maison d'écrivain... ». La Lettre de l'OCIM, ${ }^{\circ}{ }^{\circ} 125$, septembre-octobre 2009, p. 28-37 : http://ocim.revues.org/252 [consulté le 3 juin 2016].

Site de la fédération des Maisons d'écrivains : www.litterature-lieux.com [consulté le 3 juin 2016].

Sites du ministère de la Culture et de la Communication : http://www.culture.fr/Ressources/ Labels/Maisons-des-illustres/Maison-atelier-Foujita [consulté le 3 juin 2016]. 
http://www.culture.gouv.fr/culture/inventai/patrimoine/ [consulté le 29 août 2016].

\section{NOTES}

1. - Voir les sites: http://www.culturecommunication.gouv.fr/Ressources/Bases-de-donnees/ Merimee-consultable-depuis-le-moteur-Collections ; http://www.culture.gouv.fr/culture/dp/ inventaire/patrimoine/frames/index_mob.htm [consultés le 3 juin 2016].

2. - SALVY, Claude. «La maison de Foujita ». Jardin des Arts, n¹31, octobre 1961, p. 50-55.

3. - L'Apocalypse est un ouvrage monumental initié par l'éditeur d'art Joseph Forêt (1901-1991). Ce projet fut engagé en 1958, terminé en 1961. L'ouvrage reprend le texte de l'Apocalypse selon saint Jean, illustré par Bernard Buffet, Salvador Dali, Léonor Fini, Léonard Foujita, Georges Mathieu, Pierre-Marie Trémois et Ossip Zadkine. Les commentaires sont de Emil Cioran, Jean Cocteau, Daniel-Rops, Jean Giono, Jean Guitton, Ernst Jünger, Jean Rostand.

\section{RÉSUMÉS}

Entre mémoire matérielle et immatérielle, la maison d'artiste est un lieu privilégié, inspiré et inspirant. C'est un lieu intime où vie privée et espace de création s'imbriquent. Le moindre objet devient relique et la maison son écrin protecteur, de sorte que contenant et contenu s'entremêlent, au point qu'il est quasiment impossible d'envisager l'un sans l'autre. Transformer ces maisons privées en lieux publics requiert une approche délicate et une prise en compte de la forte interférence entre mobilier et bâti. Cependant, conserver l'espace en l'état fige ce qui était un lieu de vie, par nature mouvant, du temps de l'artiste. Cet état de fait pose naturellement la question du renouvellement et de l'enrichissement des collections, mais aussi celle de l'offre culturelle. Trois axes ont été définis : expliquer la trajectoire exceptionnelle d'un artiste japonais qui a choisi de vivre en France sans jamais renier ni ses racines ni son pays d'adoption, faire découvrir un atelier d'artiste préservé, enfin, donner à comprendre que, derrière une œuvre ou un objet d'art, il y a une main, une volonté, un savoir-faire.

Between tangible and intangible memories, the artist's home is a privileged place, inspired and inspiring. It is an intimate place where privacy and creational space fit together. Tiniest object becomes relics and the house becomes a protective screen, so that container and contents intertwine, to the point where it is practically impossible to consider one without the other. Transforming this private house into a place open to visitors requires a delicate approach and a consideration of the strong interplay between the furniture and the building. But conserving this space in a frozen state is in contradiction with its liveliness and changing atmospheres during the artist's lifetime. This naturally poses questions about the renewal and enrichment of the collections, but also about what exactly is on offer to the visiting public. Three axes have been defined: explain the exceptional trajectory of a Japanese artist who chose to live in France without ever denying his roots or his country of origin; discover a preserved artist's workshop; and finally, highlight artistic techniques, allowing young audiences in particular to understand that behind a painting or a work of art there is a hand, a desire, a know-how. 
INDEX

Keywords : artist's house, workshop, Foujita, Essonne, memorial site, protection, conservation, moveable assets

Mots-clés : maison d'artiste, atelier, Foujita, Essonne, lieu de mémoire, protection, conservation, patrimoine mobilier

\section{AUTEUR}

\section{ANNE LE DIBERDER}

Attachée de conservation du patrimoine, CAOA déléguée, Maison atelier Foujita alediberder@CG91.FR 